\title{
A Multi Sub-band Sliding Detection Algorithm of Ship Shaft-Rate Electric Field Signal
}

\author{
Rui Cheng ${ }^{a}$, Runxiang Jiang ${ }^{b}$ and Shenguang Gong ${ }^{c}$ \\ Weaponry Engineering College, Naval University of Engineering, Wuhan, Hubei430033, China \\ a921794197@qq.com, bjiang_runxiang@163.com, cheng-19880533@163.com
}

\begin{abstract}
Keywords: signal detection, empirical mode decomposition, shaft-rate electric field
Abstract. In order to effectively extracting out weak ship shaft-rate electric field signal immersed in strong ocean background noise, a multi sub-band sliding detection algorithm capitalizing on Empirical Mode Decomposition (EMD) with Fourth-Order Mixed Cumulant (FOMC) Diagonal Slice is proposed. Firstly, EMD method was employed to adaptively decomposition the signal into a set of Intrinsic Mode Functions (IMFs), of which the validities were determined according to correlation coefficient criterion. Subsequently exploiting the property of higher order cumulant that Gaussian colored noise can be suppressed, sliding power spectrum detection of FOMC diagonal slice of the selected IMFs was carried out. Field data processing result illustrates that, proposed algorithm can effectively and reliably detect target signal even under very low Signal to Noise Ratio (SNR), having a promising application prospect.
\end{abstract}

\section{Introduction}

Whenever a ship is sailing in the sea, a kind of extremely low frequency electric field(ELFE) generated by the modulation effect of main shaft rotation on corrosion or anti-corrosion current will be produced, namely shaft rate electric field (SREF) ${ }^{[1]}$. Both theoretical analysis and field data make it clear that SREF signal has the obvious characteristics of multiple line spectrums in frequency domain, of which the fundamental frequency in most cases locates between 1 and $7 \mathrm{~Hz}$. Owing to its long wavelength, SREF attenuates slowly with distance increasing in the seawater, thus can be used as a good signal source for remote detection of vessel target. Practically measured SREF signal having small magnitude, besides, appearance of SREF elimination devices such as Active Source Grounding system, all make SREF increasingly harder to detect. Consequently, how to effectively extract out SREF signal from strong ocean background is a problem need to be solved urgently.

To date, in the aspect of line frequency extraction and signal detection of SREF, many an algorithms have been proposed. Here we stress two typical routes. Sliding power spectrum detection algorithm being in essence a sort of energy detection method have been widely used due to its fine robustness ${ }^{[2]}$. However, performance of an energy based detection algorithm is hampered by its sensitivity to SNR. In the meanwhile, data driven signal analysis algorithms such as Wavelets ${ }^{[3]}$ or EMD adaptively decomposition signal into a set of sub-band components covering different frequency bands, making signal features more unambiguous. Besides, EMD algorithm is more superior to Wavelets in the sense that subjectivity of choosing a proper base function is eluded, making it particularly suitable for non-linear, non-stationary time series analysis ${ }^{[4]}$. Ample field data have indubitably illustrated that ocean background electric field noise obeys a complex Gaussian colored distribution form ${ }^{[5]}$, which means that higher order cumulant method can be used to reduce background noise in the sea. Inspired by the above considerations, a hybrid multi sub-band sliding detection algorithm incorporating EMD with FOMC diagonal slice ${ }^{[6]}$ is devised and tested on practically measured data in low SNRs, of which the results verify its fine performance.

\section{Algorithm Procedure}

Suppose $t_{w}$ (s) be data window length, $t_{d}$ (s) be detection window length, $t_{i}$ (s) be sliding step, sampling rate $f_{s}(\mathrm{~Hz}), M$ be the number of sampling points in frequency domain. Assuming $x_{n}$ be 
the sensor output value in time instant $n$, then the data series to be processed is denoted as $X(n)=[x(n), x(n-1), \cdots x(n-N+1)]$, while $N=t_{w} \times f_{s}$ being the number of points in the data window. Here a sliding detection algorithm of ship SREF signal combing EMD with FOMC diagonal slice is proposed, of which implementing steps are as follows.

(1) Exploit EMD method to decompose data series to be processed in specified data window at time instant $n$, getting several IMF components and a residual term;

(2) Calculate corresponding correlation coefficient (CC) values between every IMF and source signal, screen out effective IMF by the criteria that the CC value is greater than $e(0<e<1)$ times of correlation coefficient maximum, denoted as $\left\{i m f_{l}, l=[1, \cdots L]\right\}$ with $L$ being the number of effective components;

(3)Using Periodogram method, $M$ point power spectrum of FOMC diagonal slice of IMF component $\mathrm{imf}_{l}$ is computed, of which the mean value is considered as eigenvalue at time instant $n$, denoted as $p_{n, l}$;

(4) Compute threshold value at time instant $n$ in sub-band $i m f_{l}$, which is equal to $u$ times the mean value of power spectrum eigenvalues of all the time instants within detection window. That is to say, if $p_{n, l}>h_{n, l}=u \times \frac{p_{n-1, l}+p_{n-2, l}+\cdots p_{n-w+1, l}+p_{n-w, l}}{w}$, while $w=t_{w} \times f_{s}$ is number of time instants in the detection window, target signal existence is confirmed once at time instant $n$ at sub-band $\operatorname{imf}_{l}$;

(5) Slide forward in time in accordance with step $t_{i}$, repeat steps (1) to steps (4) above, calculate eigenvalue $p_{n+1, l}$ and threshold $h_{n+1, l}$ at next time instant $n+1$ of component $i m f_{l}$. When target existence are confirmed consecutively $K$ times, target signal existence can be determined at time instant $n+K$ in sub-band $i m f_{l}$;

(6) Take all the time instants confirming existence of target signal into consideration altogether. As to a certain time instant, if in more than $R(0<R \leq L)$ sub-bands algorithm confirms target signal existence, finally we can say the target signal does exist at this time instant.

Algorithm begins to work from time instant $t_{w}+t_{d}$, and thanks to floating threshold, has good anti-interference performance, which is vital to SR signal detection in practical ocean environment. Parameters $e, u, K, R$ should be decided properly based on the SNR condition. When SNR is relatively in good condition, EMD method extracts signal energy very well, a small $e$ will prevent unwished noise from being included. In the meanwhile, big $u, K, R$ value will reduce false alarm rate. On the contrary, a big $e$ together with small $u, K, R$ can improve the detection sensitivity. Moreover, threshold value at time instant $n-\Delta n$ can be taken as threshold at time instant $n$ to eliminate the neighboring effect at a given detecting time instant furthermore.

\section{Example Verification and Result Analysis}

Utilizing homemade inner-recording measurement system located at the sea bottom, we obtained lots of practical vessel SREF signal and ocean background noise. To a large extent, these data benefit us in investigating into signature feature of SREF signal and finding a proper algorithm to reliably and sensitively detect vessel targets in the sea. Measurement condition parameters during measurement period were: water depth about 20 meters, sediment texture in the sea bottom, wave scale being between Level 1 to 2, sampling rate was $20 \mathrm{~Hz}$. To verify the validity of proposed algorithm, several sets of mixed data with different SNRs were subjected to sliding detection test. Relevant Algorithm parameters were set as $t_{w}=20, t_{d}=50, t_{i}=1, M=1024, e, u, K, R$ changing with SNRs. 
Field data of SREF signal belonging to certain ship and ambient ocean noise are shown in Fig.1, represented in both time domain and frequency domain. Normalized Power spectrums were estimated by means of periodgram method. From Fig.1, we can clearly see that SREF signal features a multiple harmonic frequency structure, while the ambient noise does not have obvious line spectrum. Besides, owing to the attenuation effect of conductive seawater on high frequency electromagnetic wave, power magnitude decreases sharply with frequency increasing.
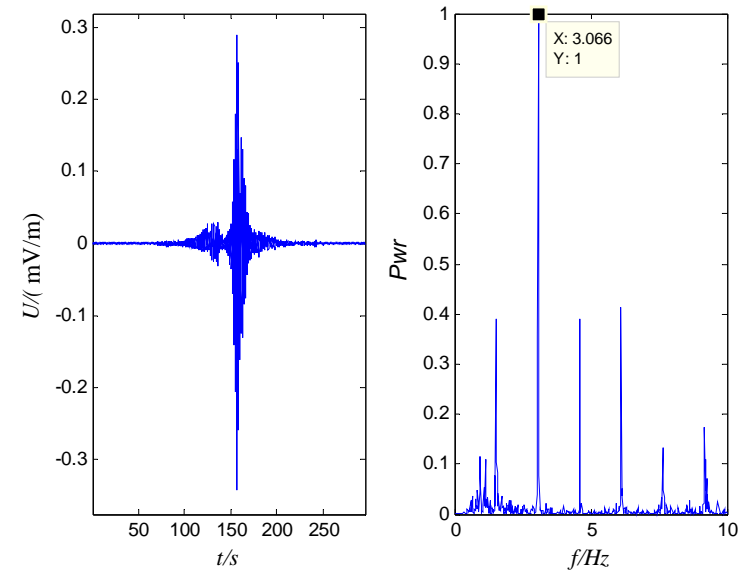

(a) SREF signal
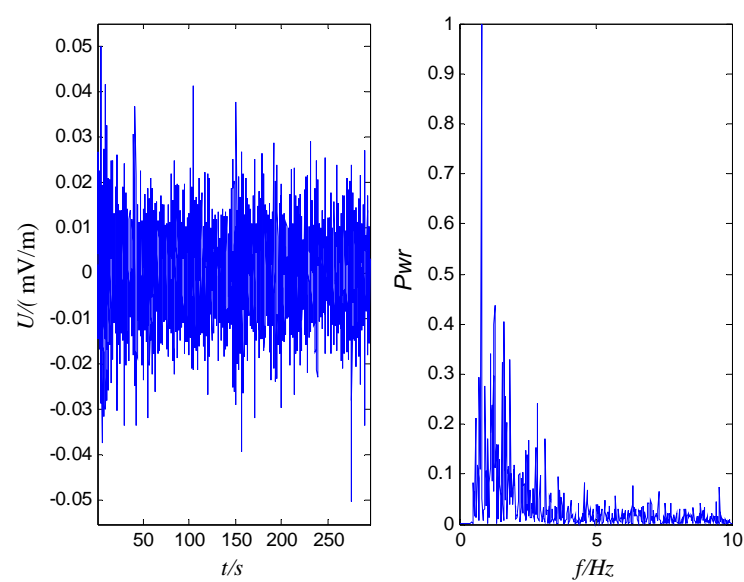

(b) Ocean Background Electric Field

Fig.1 Example of SREF Signal and Environmental Noise

During the measurement, sea state is relatively good, thus SREF data under low SNRs have not been acquired. To examine performance of proposed algorithm in the context working under low SNRs, mixed data are generated by considering SREF and background in Fig. 1 to be "pure signal" and "pure noise". Simulation is based on amplitude linear superposition according to specified SNR, of which is defined as follows:

$$
S N R=10 \lg \frac{E_{s}}{E_{n}}
$$

$E_{s}$ and $E_{s}$ denote SREF and background noise amplitude respectively.

Oscillogram and power spectrum graph of mixed signal with SNR equaling to $-15.4 \mathrm{~dB}$ are shown in Fig.2. As clearly shown in the figure, SREF signal cannot be identified out either in time domain or in frequency on account of no obvious distinction between signal existence and signal absence.
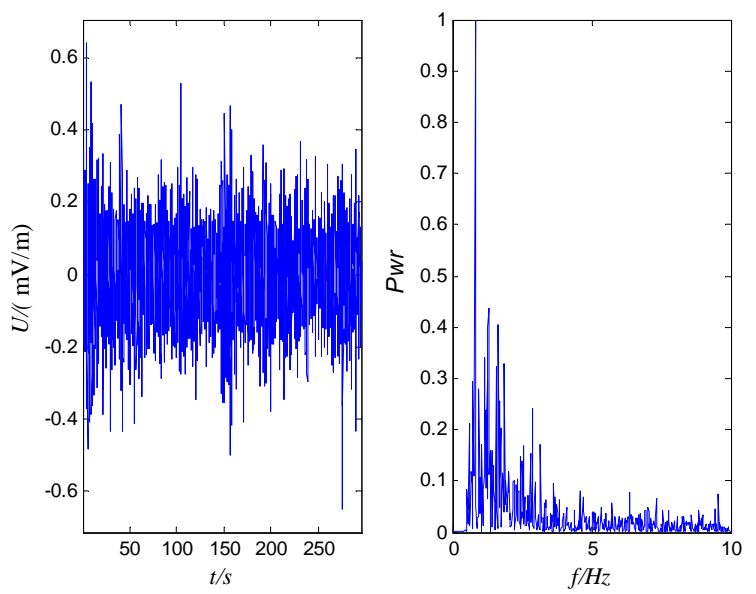

Fig.2 Mixed Signal illustration

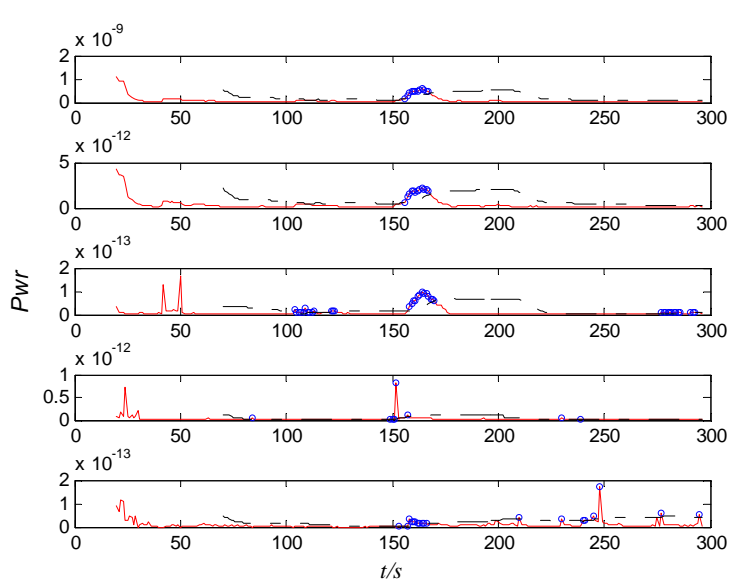

Fig.3 Detection graph of the proposed Algorithm

Processing results of proposed algorithm are shown in Fig.3. From top to bottom are detection graph of mixed signal itself and all the four effective IMFs respectively. $e=0.4, u=3, K=3$, $R=2$. Target signal existence is confirmed at 159s, which was just about the moment when signal reaches its peak value. 
After a comprehensive analysis of a mass of numerical experiments based on field data with different SNRs, several conclusions can be reached.

(1) A method that combines FOMC with sliding detection, taking advantage of Gaussian colored noise suppression ability of the former and robustness and stability of the latter ,can still pick out target signal accurately when traditional sliding power spectrum detection algorithm fails;

(2) EMD algorithm fulfills adaptive signal decomposition, and with redundancy brought by modal aliasing incidentally, constitutes multi sub-band detection, improving algorithm's reliability.

(3) Specifically, as can be seen from Fig.3 above, the second IMF component confirms signal existence between 106s and 113s, far ahead of 159s. Looking back at Fig.1 (a), this time span corresponds to front "small envelop" of signal wave profile. This implicitly manifests the feature of EMD algorithm that it can extract local time scale characteristics of analyzed signal.

\section{Conclusions}

In the context, a sliding detection algorithm combining EMD, effective IMF selection and FOMC diagonal slice power spectrum estimation applied to SREF signal identification is proposed. Through EMD the signal is adaptively decomposed into multiple sub-band components, of which FOMC power spectrum is slidingly detected. In the end all the sub-band detection results are fused altogether to give a final judgment. Processing results with field data during sea trial have demonstrated fine performance of the proposed algorithm.

\section{Acknowledgements}

This work was financially supported by the National Natural Science Foundation under Grant 51109215 and 51509252.

\section{References}

[1] S.Li, C.H.Zhang, J.D.Luan: Acta Armamentarii Vol.30 (2009), p.1738-1742 (in Chinese)

[2] H.Tan, Z.H.Bao, S.G.Gong: Journal of Naval University of Engineering Vol.23 (2011), p.96-99 (in Chinese)

[3] Y.Z.Jia, R.X.Jiang, S.G.Gong: Journal of Huazhong University of Science and Technology (Nature Science Edition) Vol.41(2013),p.25-29(In Chinese)

[4] Information on https://www.clear.rice.edu/elec301/Projects02/empiricalMode/

[5] P.Hu, S.G.Gong, Y.D.Hu: Journal of Huazhong University of Science and Technology(Nature Science Edition) Vol.39(2011),p.15- 18(In Chinese)

[6] A.Massoud, A.Osman and A.Noureldin: Computers \& Electrical Engineering Vol 38(2012), p.986-993. 